\title{
Introducing GIS-based simulation tools to support rapid response in wildland fire fighting
}

\author{
A. Moreno ${ }^{1}$, A. Segura ${ }^{1}$, S. Zlatanova ${ }^{2}$, J. Posada ${ }^{1}$ \\ \& A. García-Alonso ${ }^{3}$ \\ ${ }^{1}$ Vicomtech Research Center, San Sebastián, Spain \\ ${ }^{2}$ Delft University of Technology, Delft, The Netherlands \\ ${ }^{3}$ University of the Basque Country, San Sebastian, Spain
}

\begin{abstract}
Once a forest fire is detected, decision makers have to evaluate the situation in order to mobilize all the required resources in the most efficient way. This work presents how a GIS-based simulation tool provides valuable information to the decision makers, so they can trigger rapid and efficient instructions to the individual brigades and fire fighters. The capabilities of the tool allow running independent simulations under hypothetical situations and what-if scenarios, so decision makers can analyze the impact of different actions on a virtual scenario. Keywords: decision making software, decision support system, real time simulation, forest fire spread simulation, GIS-based tool.
\end{abstract}

\section{Introduction}

Emergency Management field comprises different disciplines, concepts and actors. It is oriented towards the management of the resources and the workforce to protect the society from hazard risks provoked by catastrophic events.

In the case of eventual forest fires, Emergency Management triggers the corresponding protocols and procedures, including the mobilization of the local, regional or even national authorities (Civil Protection) and entities (hospitals, police stations, fire stations...). All the actors involved in the task are coordinated from the designed Command Control where all the tactical and logistic decisions are made.

A rapid response after the detection of a new forest fire is desirable for multiple reasons, but essentially, it is important to fight and contain the effect of 
the forest fire. Rapid fire fight diminishes human safety risks and reduces the environmental and economic impact of the fire.

Although the official protocols differ between cities, regions and countries, there are some core techniques and methodologies common to all of them. One of the first steps is to get as much information as possible, including the geographical information, the weather status, the road network status and the knowledge about the available human and technical resources.

Once all the preliminary information is collected and processed, a first set of immediate decisions are made following the official protocols. This work presents how a GIS-based simulation tool provides valuable information to the decision makers, so they can trigger rapid and efficient instructions to the individual brigades and fire fighters. The simulator capabilities enable visualizing and assessing how the forest fire spreads in wild-land zones, using a very fast algorithm to update a cell-grid which represents the forest area.

In the next section some related work will be presented, followed by a brief description of the capabilities of the GIS-based fire fighting simulation system. That section also presents how this fire fighting simulator might be used in the Decision Making Process to get valuable information, using two distinct scenarios.

\section{Related work}

The Decision Making Process (DMP) [1] is a complex process, which starts with a question, a problem or a situation and may result in a solution for it, optimal or not. After collecting and analyzing the available information about the issue, the decision makers evaluate all the possible options, and through a mixture of analytical processes, expertise and intuition, the most suitable solution is adopted.

Essentially, any Decision Support System (DSS) is divided in three main components: the KnowledgeBase, the Model and the User Interface. DMP requires an information system where data and previous experiences are stored and queried back.

Pandit and Zhu [2] proposed the utilisation of Semantic technologies to integrate heterogeneous systems in a domain. An ontological framework supports the DMP by means of the generation, analysis, sharing and reuse of the domain knowledge. Finally, the User Interface is presented to the users through Decision Making Software (DMS), aimed to present the data to the users, so they can easily overview the data and take the best decision based on any criteria using techniques coming from the Multi Criteria Decision Analysis (MCDA).

Temiz and Tecim [3] presented a spatial MCDA system applied to the planning phase of the forest fire fighting. Different criteria are studied, analysed and visualized in $2 \mathrm{D}$ coloured drawings, where most relevant information is clearly highlighted. However, the methodologies lack any kind of GIS support.

DigitalFusee [4] is a web service aimed to support the creation of training exercises using virtual fires. It shows, in a $3 \mathrm{D}$ environment, the effects on the fire 
behavior caused by terrain geomorphology and weather changes. The system uses Bing Maps to retrieve the elevation maps, Landfire to get the land use of a given area and Farsite to run the fire simulations (see Fig. 1, left).

Xioapeng et al. [5] presents a GIS-based tool to visualize Jiaohe city in a 3D environment. It combines a 3D terrain with other geo-information as roads, water bodies and land use. The tool also connects with external sources to get real time information (GPS or remote sensors), visualizing the information in the 3D environment. The users have several useful tools to get custom information to help in DMP (see Fig. 1, right).

Premfire [6] provides an integral solution for monitoring forest fire, with three modules. The module $C C$ is the Command and Control tool, where all relevant information is presented to the managers (alerts, resources, cartography and reports). Mobile (see Fig. 2) is the module used by the fire and brigade chiefs
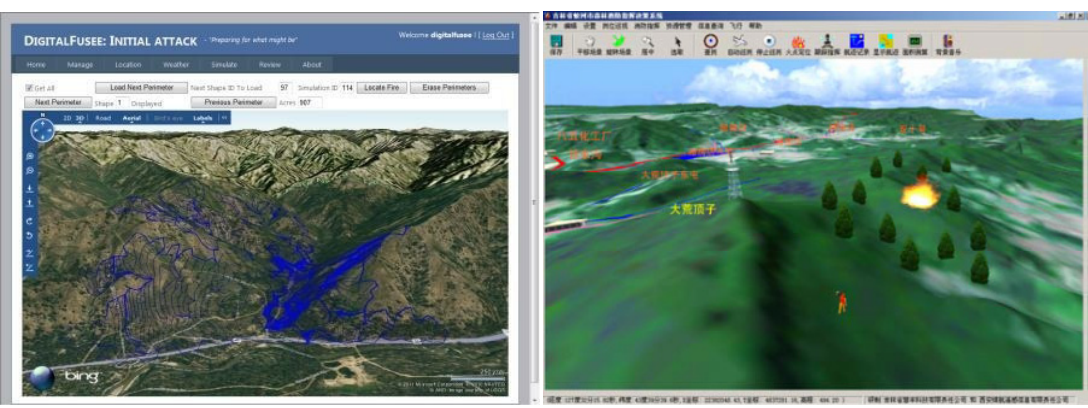

Figure 1: Left: DigitalFusee [4] uses Farsite kernel to get the fire spread simulation. Right: 3D visualization of a forest fire, with the road network and human resources [5].

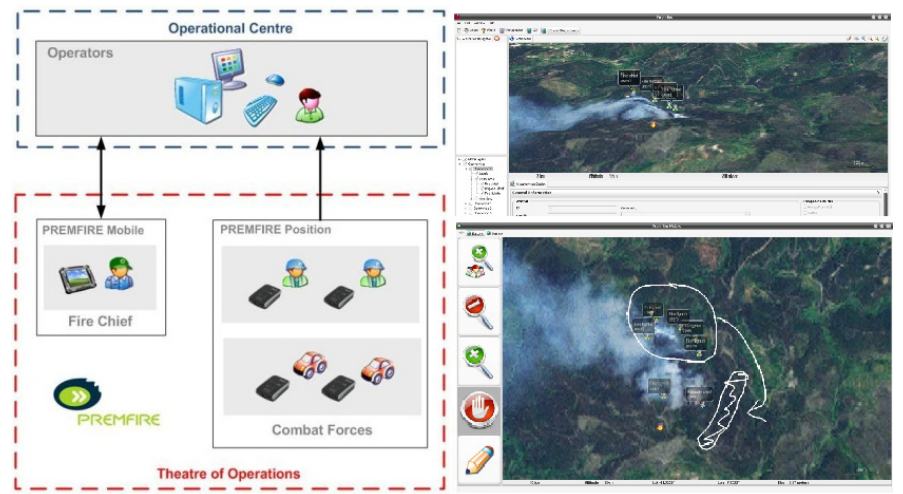

Figure 2: Premfire command and control architecture and examples of Premfire mobile [6]. 
to communicate with the Command and Control tool. It allows real time tracking and visualization of the resources in a $3 \mathrm{D}$ environment and helps in taking decisions about reallocating resources. Mobile also gathers the GIS information and keeps it updated during an emergency. The Position module is focused in real time tracking of the fire workers on the field, communicating their positions to the $C C$ and Mobile modules.

Summarising, most of the current simulation tools aimed to support the DMP lacks versatility in the early stages of an emergency. These tools use well stabilised simulation kernels, characterised by their accuracy, but their non real time performance limits its utilisation. Our work also addresses the integration of GIS data in the early stages of emergencies.

In the next section, a fast and approximated forest fire spread simulator is presented. The utilisation of the results in the DMP for rapid response in fighting forest fires is analysed through two different scenarios.

\section{Methodology}

The utilisation of geo-referenced tools in the earlier phases after a fire is detected provides useful information to the decision makers. In this section, we present how a GIS-based fire fighting simulator is able to obtain a very rapid and approximated simulation of the evolution of the fire, helping to make decisions in this critical initial phase of a forest fire.

In the following subsections, a brief introduction to the common functionality of the simulator will be addressed, followed by the explanation of the scenarios where the simulator will show the advantages of the utilisation of such tool. The focus of this paper is on the connection with GIS information (sections 3.1 and 3.3) and assessing the versatility offered by these tools (sections 3.2, 3.4 and 3.5). The detailed description of fire propagation/extinction algorithms (section 3.1) and their validation is dealt in other works [7].

\subsection{Fire fighting simulator capabilities}

The GIS-based fire fighting simulator is oriented to get rapid results for the fire fighting procedures, in forest and urban areas. The system (Fig. 3) must provide a fast response to allow users to test how changes in the environment or human actions may affect fire propagation/extinction. Some fire spread and exhaustion algorithms have been modelled and developed [7], in order to meet this real time interaction performance requirement. The very well known and accurate fire spread algorithms existing in the bibliography $[8,9]$ cannot meet the required fast response.

The fire spread simulations take static data as input. In runtime, dynamic data is continuously generated or updated by the simulation itself (state of the fire spread), by external modules (weather conditions) and user actions (e.g. add extinguisher).

The most important static information is the topographic information of the scenario where the fire simulation will be held, which includes the terrain itself 


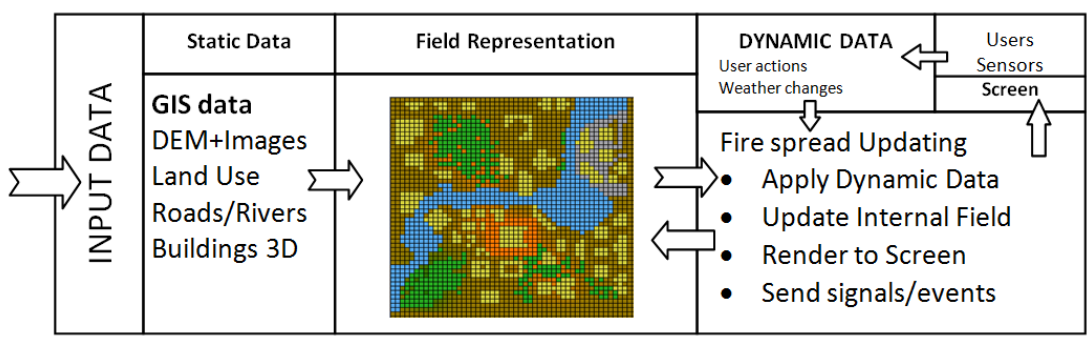

Figure 3: Architecture of the fire fighting simulator related to the GIS data and the field updating mechanism.

given as a Digital Elevation Model (DEM), the aerial imagery and the ground classification (land use), given by disjoint zones and normally coming from GIS systems [14] (Table 1). Although detailed information would be great, at least a rough classification based on Water Bodies (including sea, lakes and rivers), Roads, Urban Areas and Forest Areas is required (see Fig. 4). Some data sources like natural or artificial barriers (mountain roads or firewalls) are not easily accessible for the public, as they are not commonly represented in the maps. In these cases, it would be required to ask to the corresponding local authority for such information, sometimes represented in traditional paper maps.

The dynamic information represents all the variables calculated in runtime like amount of fire at a given point; the amount of remaining fuel, since it could be partially burnt; or the remaining active extinguisher, thrown by a fire fighter, a truck or an aircraft.

The weather conditions are considered as dynamic data too, although the information comes from external modules.

Input information is pre-processed and sampled into the internal field representation (see Fig. 3), based on a grid of autonomous cells, each of them containing geometrical information, such as position, dimensions, elevation and slopes [15].

Table 1: $\quad$ Summary of the some geo-information providers for the VR fire fighting simulator.

\begin{tabular}{|c|c|c|}
\hline GIS Data & Data Sources & Details \\
\hline \multirow{2}{*}{ Cartography } & SRTM [10]/ASTER [11] & Low Resolution. Global \\
\cline { 2 - 3 } & Regional Aerial Scans & High resolution. Local \\
\hline \multirow{2}{*}{ Imagery } & - & Height as False Colour \\
\cline { 2 - 3 } & Regional Aerial Imagery & $\begin{array}{c}\text { High resolution in } \\
\text { Regions (LIDAR, DEM) }\end{array}$ \\
\hline Land Use & CORINE Land Cover [12] & Highly detailed \\
\hline Roads & OpenStreetMap [13] & Collaborative community \\
\cline { 2 - 3 } & Commercial products: GPS & Highly detailed, updated \\
\hline Firewall in mountains & Local Authorities & Sometimes no digital info \\
\hline
\end{tabular}



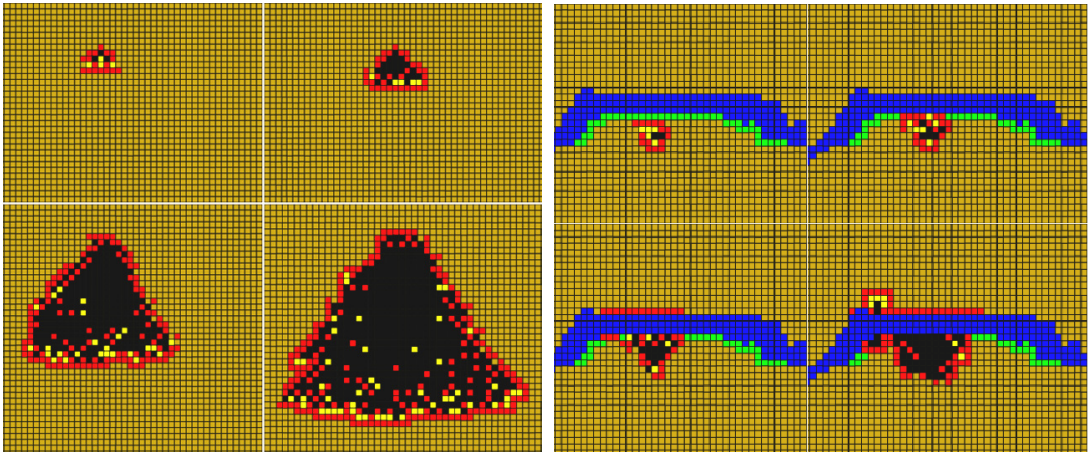

Figure 4: Evolution of a forest fire on flat terrain. Left: North wind. Right: South wind allows fire to jump over the river.

Although other parameters like humidity and global temperature are relevant for an accurate simulation, the wind speed and direction are considered the most influent variables [15], as they modify the fire behaviour in a sensible way. The implementation of the radiation effect simulates how the fires spread across a barrier (river, road or firewall) if the wind conditions are favourable.

The simulator supports the extinction procedures by means of throwing water resources to the field. The high level implementation allows the emulation of the behaviour of the fire fighters with manual hoses; fire trucks or even aircrafts (see Fig. 5).
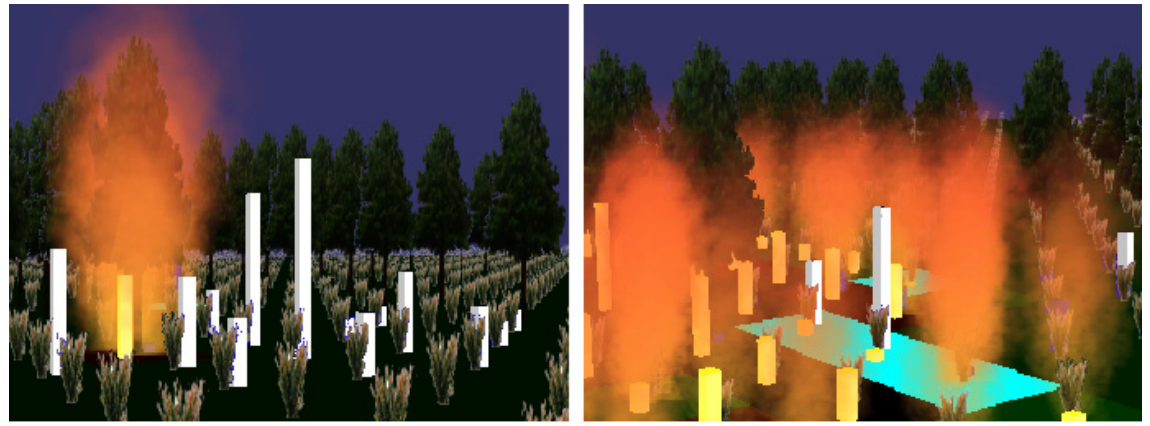

Figure 5: Forest fire extinction by adding water resources to the field. White bars represent the amount of water on the cell, while yellow bars represent the intensity of the fire.

\subsection{Utilisation for rapid response simulations}

In the presented GIS-based fire fighting simulator, the resources on the field can be added, edited or removed, affecting almost immediately to the results of the ongoing simulation.

This versatile functionality of the simulator allows decision makers the visualization and configuration of different hypothetical situations. These fast 
analyses can be used to improve the organization of the human resources and their equipment. All this collected information adds important knowledge in the DMP.

In the scope of this work, we have considered two main strategies to use the GIS-based fire fighting simulator in the early stages of a detected forest fire. The first one is to configure the virtual scenario and run independent simulations using real time information coming from the field, following the instructions of the experts to set up the fire resources. The second is aimed to help decision makers to evaluate the outcome of their actions under what-if conditions, where instead of using actual data, other hypothetical situations can be analyzed in order to be prepared to affront possible hazards, for instance, wind changes.

Both strategies need access to geographic data, so the virtual scenario can be constructed by the simulator kernel. Among all the available GIS data, the minimum subset includes the elevation information, so the terrain slopes could be calculated; the land cover use, including water bodies and rivers; and the road network. In a similar way, at least, the wind parameters are required by the simulator. Any other weather variable, although important, is less relevant than the wind speed and direction [15].

\subsection{Loading information into the simulator}

In the case of a recently spotted forest fire, the first task is to collect all the data related to the location and status of the forest fire. It is reasonable to assume that a general GIS should be easily accessible, but also it is required to get some updated and real time geo-information about the status of the road network, the weather and other important measurements, normally linked in the specific protocols for Emergency Management [16].

The simulation system enables the integration of heterogeneous GIS data sources into the simulation scenario. When a forest fire is detected, the simulator is fed with the geo-information of the zone where the fire is located (digital elevation maps, land use, vegetation classification, road network, barriers, water masses...) including the existing residential constructions that might be scattered in this area.

In this work, the simulation platform has been implemented using OpenSceneGraph [17] graphical API as the backend rendering engine, a high performance real time graphics library based on OpenGL. The terrain model for the targeted zones has been generated as a multi-resolution 3D model, using VirtualPlanetBuilder to convert the geospatial imagery and elevation data to the terrain 3D model.

The OpenSceneGraph's native support for Esri Shapefiles (SHP) has been used to load into the virtual scene the roads, buildings and other geographical information. Additionally, that very same functionality has been adapted to convert the land use information, provided as shapefiles, to our internal representation of the field.

Once all the static information is loaded into the simulator, the weather parameters are retrieved from an XML file where a global wind direction and 
strength and per tile wind information can be loaded into the virtual field (see Fig. 6).

In our particular scenarios, we have used the public data provided by the Basque Government through the OpenData [18] initiative to build the 3D virtual world and the real time weather data provided by EUSKALMET, the Basque Meteorology Agency [19].

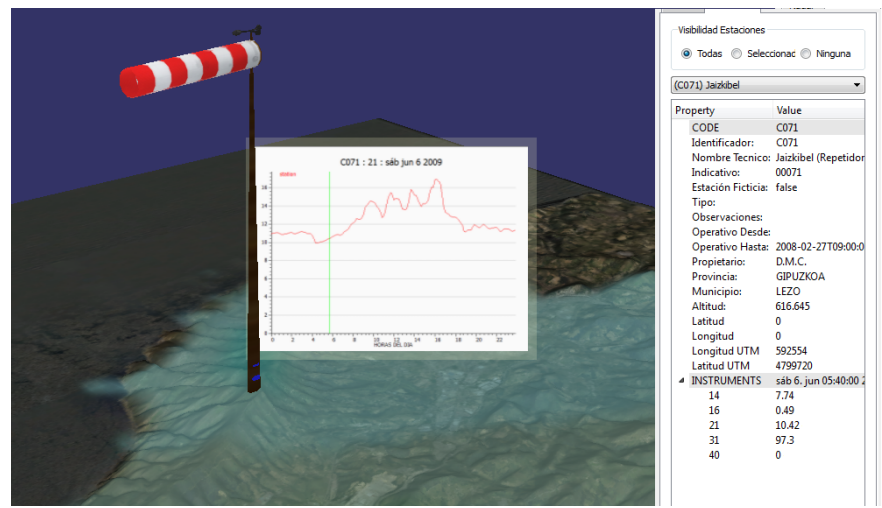

Figure 6: 3D GIS visualization with temperatures texture overlay, daily temperatures in $2 \mathrm{D}$ and $3 \mathrm{D}$ visualization of wind speed and direction using a virtual windsock.

\subsection{Scenario 1: Run simulations with continuously updated information}

The first scenario results in standalone simulations of the evolution of the forest fire. The speed and efficiency of the used methods can iteratively obtain the stepby-step tiled representation of the fire in a matter of seconds for a real period of one hour with a time step of 10 seconds, i.e., around 360 simulation steps in less than one minute in a dual-core PC.

The efficiency of the simulation depends on the number of active tiles. However, at the very beginning of a forest fire there are not many, so the simulation steps are very fast to calculate.

While any forest fire is active, people on the field, the meteorological agencies and other measurement equipment provide constantly updated information to the Control Centre. Feeding this new information to the fire fighting simulation is quite straightforward, and therefore, new and more accurate simulations can be obtained.

These updated simulation results can lead to modifications in the previous tactical decisions on the field, in order to try to be more effective and productive against the forest fire. In this scenario, technicians will be responsible to import newly arrived data so the decision makers can get new results coming from the GIS-based forest fire spread simulator. 
Even without the addition or configuration of the fire fighting resources, the new simulations obtained in a matter of seconds will provide precious information about the expected fire spread.

\subsection{Scenario 2: What-if branching}

Golfarelli et al. [20] defined what-if analysis "as a data intensive simulation whose goal is to inspect the behaviour of a complex system".

In our forest fire domain, this what-if methodology can be applied to the input data of the simulations, obtaining different and hypothetical results. A proper selection of the what-if branching can obtain a set of results, which under the criteria and scrutiny of the decision makers could help to fight the ongoing forest fire and reduce human hazards.

Schuman and Rohrbaugh [21] described four types of what-if questions and their meaning. Applied to the fire fighting scenario, the questions can be rephrased as:

- If the resources are deployed in this way, what results?

- If the wind conditions change, what results?

- If the results from different configurations and conditions are collected, which one is preferred?

The first question is supported by the simulator, as the water resources can be placed and configured interactively on the virtual field.

The second question suggests to check what would happen if the wind conditions change at a given time, or some kind of extreme wind condition analysis to check the worst expected scenario.

The last question addresses the issue of presenting all collected information in all the branches. This huge amount of information may help the decision makers to choose the most suitable actions.

Theoretically, all the actions on the field can be branched, but this could lead to a non affordable number of parallel simulations. So, the users will have to decide which of their actions will be branched in the simulation and under what conditions. For example, it could be interesting to know what would happen if a brigade with manual hoses is placed in a given position or another, but arriving 10 minutes later (see Fig. 7).

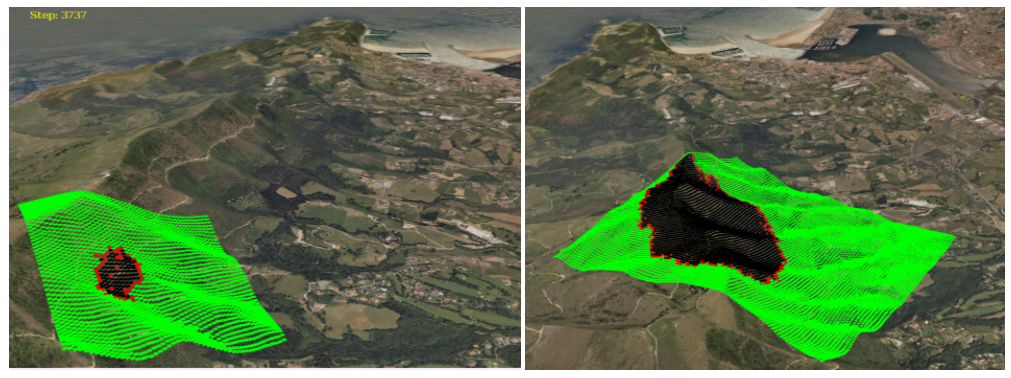

Figure 7: Expected evolution of a forest fire with a strong NE wind (right) opposed to current wind conditions. 
Even with a very small number of branches, the number of hypothetical situations could be very high. So, it is recommended to be focused on a very small set of conditions, keeping the number of branches under control.

Additionally, as new real data come from the field, the previous simulation results would be nullified and therefore, new simulations, including the possible branches, should be run again or, at least, their conditions should be reconsidered by the decision makers.

\section{Conclusions and future work}

In the Emergency Management, it is rather important to get all the available information as soon as a new emergency is detected. This work has presented how a GIS-based fire fighting simulator can be introduced in the Decision Making Process after a forest fire is detected.

The design and implementation of the simulator have been oriented to get fast simulation results of the forest fire spread [6], allowing the interactive modification of the elements in the virtual field. The advantages of such rapid simulation enable its use in the early stages of a forest fire.

This work has presented two scenarios where the GIS-based forest fire simulator produces valuable information to the decision makers, so they can try to fight the forest fire in the most efficient and safest way. Using the proposed tools and approach, more analytical scenarios could be provided to the decision makers. For example, simulations under extreme wind conditions and in different directions could be run, so the decision makers could have information about the potential risky areas and take early decisions.

The simulation results are not stored so all the decisions, the branching conditions, the field and other valuable knowledge is lost. In the future, the simulation results and the decision taken could be introduced in a Semantic powered tool, so the experiences could be stored, queried and retrieved. The decisions and the results of the decisions would be added to the system, being available for further analysis, after the forest fire is extinguished or as Knowledge Base in similar incidents.

\section{Acknowledgements}

This work was supported by COST Action TU0801 "Semantic Enrichment of 3D City Models for Sustainable Urban Development", and it was carried out in the context of project SIGEM, funded by the Spanish Industry Ministry through its Avanza I+D Programme. Dr García-Alonso was partially supported by the Spanish Government (TIN2009-14380) and the Basque Government (B66 April $22^{\text {nd }} 2010$ and UE09+/32). 


\section{References}

[1] Finlay, P., Introducing decision support systems. Blackwell Publishers, Cambridge, Mass, 1994.

[2] Pandit, A. and Zhu, Y., An ontology-based approach to support decisionmaking for the design of ETO (Engineer-To-Order) products. Automation in Construction. Vol. 16 (6). 759-770, 2007.

[3] Temiz, N. and Tecim, V., Spatial Multi Criteria Decision Making in Forest Fire Fighting Planning. Endüstri Mühendisliði, 20(2), pp. 15-30, 2009.

[4] DigitalFusee: Wildland Firefighter Training, http://digitalfusee.com

[5] Xiaopeng, W. and Gaofeng, K. and Xiaobin, H., Forest Fire Fighting Command and Decision Making System Study based on 3S Technologies Take Jiaoche City in Jilin Province as an Example. The International Archives of the Photogrammetry, Remote Sensing and Spatial Information Sciences. Vol. XXXVII. Part B4. pp. 1113-1116, 2008.

[6] Premfire: Emergency Command and Control. http://www.premfire.net/.

[7] Moreno, A. and Segura, A. and Korchi, A. and Posada, J. and Otaegui, O., Interactive urban and forest fire simulation with extinguishment support. Advances in 3D Geo-Information Sciences, Lecture Notes in Geoinformation and Cartograph, Springer Berlin Heidelberg. 131-148, 2011.

[8] Rothermel, R., A mathematical model for predicting fire spread in wildland fuel. Tech. rep., U.S. Department of Agriculture, Forest Service, Intermountain Forest and Range Experiment Station, 1972.

[9] Finney, M., Farsite: Fire area simulator-model development and evaluation. Res Pap RMRS-RP-4 Ogden, UT: USDA Forest Service, Rocky Mountain Research Station 1:47. 1998.

[10] Shuttle Radar Topography Mission. http://www2.jpl.nasa.gov/srtm/

[11] ASTER: Advanced Spaceborne Thermal Emission and Reflection Radiometer. http://asterweb.jpl.nasa.gov/

[12] CORINE Land Cover, Commission of the European Communities. 1994. http://www.eea.europa.eu/publications/COR0-landcover.

[13] OpenStreetMap, collaborative map. http://www.openstreetmap.org/

[14] Dumond, Y., Forest fire growth modelling with geographical information fusion. Information Fusion, 11th International Conference. 1- 6, 2008.

[15] Weise, D.R. and Biging, G.S., Effects of wind velocity and slope on flame properties. Can J For Res, 26, pp. 1849-1858, 1996.

[16] Zlatanova, S., Formal modelling of processes and tasks to support use and search of geo-information in emergency response, Proceedings of the 13th International Conference and Exhibition on Geospatial Information Technology and Applications, 2010.

[17] OpenSceneGraph: Open source 3D Graphics API over OpenGL. http://www.openscenegraph.org/.

[18] OpenData Euskadi programme. http://opendata.euskadi.net

[19] EUSKALMET, Basque Country Meteorology Agency. http://euskalmet.net/ 
174 Modelling, Monitoring and Management of Forest Fires III

[20] Golfarelli, M. and Rizzi, S. and Proli, A., Designing What-if Analysis: Towards a Methodology. DOLAP'06: Proceedings of the 9th ACM international workshop on Data warehousing and OLAP. 51-58, 2006.

[21] Schuman, S. and Rohrbaugh, J., Four what ifs? Evaluation of alternative technologies for the destruction of chemical weapons. Journal of Policy Analysis and Management, 23, pp. 901-908, 2004. 